\title{
Expansión de un sistema de transmisión mediante LOPF-AC
}

\section{Expansion of a transmission system using LOPF-AC}

DOI: https://doi.org/10.17981/ingecuc.14.2.2018.11

Artículo de Investigación Científica. Fecha de Recepción:19/05/2018. Fecha de Aceptación:12/12/2018.

\author{
Pablo Escudero-Delgado \\ Universidad Politécnica Salesiana. Quito (Ecuador) \\ poul08_es@homail.com \\ Diego Carrión-Galarza \\ Universidad Politécnica Salesiana. Quito (Ecuador) \\ dcarrion@ups.edu.ec
}

Para citar este artículo:

P. Escudero-Delgado y D. Carrión-Galarza, "Expansión de un sistema de transmisión mediante LOPF-AC," INGE CUC, vol. 14, no. 2, pp. 116-125, 2018. DOI: http://doi.org/10.17981/ingecuc.14.2.2018.11

\section{Resumen}

Introducción- En la presente investigación se transforma las ecuaciones que conforman un OPF-AC a un sistema de restricciones lineales mediante series de Taylor, por lo cual se adquiere un modelo LOPF-AC, preciso y aplicable para poder garantizar la minimización de pérdidas en todo el sistema.

Objetivo- Minimizar las pérdidas en la expansión del sistema de transmisión.

Metodología- Se basa en linealizar las ecuaciones del OPF-AC mediante Series de Taylor, para obtener un problema linealizado.

Resultados- El modelo determina cuales son las líneas que se deberían implementar y cuales se deberían reforzar, considerando el menor costo y la minimización de las pérdidas.

Conclusiones- La demanda total de la red más la proyección de carga de los distintos casos para la expansión del sistema de transmisión es abastecida con normalidad cumpliendo con los parámetros establecidos de generación y transmisión conjuntamente con las restricciones del algoritmo para obtener un desempeño óptimo en la TEP.

Palabras clave- Flujos óptimos de potencia, Linealización, Minimización de pérdidas, Planificación de la expansión de la Transmisión, Sistemas eléctricos de potencia.

\begin{abstract}
Introduction- In this document we transform the OPF$\mathrm{AC}$ equations into a system of linear constraints using Taylor series, for which a LOPF-AC model is acquired, accurate and applicable to guarantee the minimization of losses in the whole system.
\end{abstract}

Objective- To minimize the electrical losses in the expansion of the transmission system.

Methodology- It is based on linearizing OPF-AC equations by Taylor series, to obtain a linear problem.

Results- The model determines which lines should be implemented and which ones should be reinforced, considering the lower cost and the minimization of losses.

Conclusions- The total demand of the network plus the loading projection of the different cases for the expansion of the transmission system is supplied normally, complying with the established parameters of generation and transmission together with the constraints of the algorithm to obtain an optimal performance in the TEP.

Keywords- Electrical power system, Linearization, Losses minimization, Optimal power flow, Transmission expansion planning. 


\section{INTRODUCCIÓN}

La planificación de la expansión de transmisión (TEP), aborda el problema de aumentar las líneas de transmisión de un sistema de potencia existente. El objetivo es abastecer de manera óptima a la carga eléctrica. En general, se considera que la TEP toma una decisión estocástica sobre la hora, ubicación y qué tipos de líneas de transmisión se instalarán [1], [2] especially in an oligopolistic electricity market in which a number of strategic (price-maker; aspecto por el cual, se debe hacer una correcta planificación de la expansión de las líneas candidatas para el sistema, teniendo en cuenta la inquietud de solventar los problemas relacionados con la demanda y la sostenibilidad del sistema [3], [4],[5] by means of which an optimal flow of AC power (OPF-AC.

Para la expansión, se debe prever que la red de transmisión existente no es capaz de transferir toda la generación eléctrica total, entonces esto implica determinar dónde y cuántas líneas candidatas deben agregarse al sistema existente, al menor costo tomando en cuenta valores de demanda futuros [3], [6], [7].

La TEP consiste en determinar el plan de inversión que se necesita para poder reforzar el sistema de transmisión, con el fin de tener costos y pérdidas mínimas en las líneas de transmisión (LT). Por tal razón, es considerado como un problema de optimización, ya que se debe minimizar factores que son importantes para tener un mayor rendimiento de la red a estudiarse. Por otro lado, el modelo de flujo de potencia de TEP basado en AC tiene varias ventajas [8], tales como: a) permite al planificador continuar con otros estudios después de resolver el problema de TEP [9], [10]; b) la posibilidad de usar los dispositivos de operación no lineales para resolver el problema; c) tiene la capacidad de llevar a cabo la etapa de refuerzo cuando se utilizan los planos obtenidos a partir del modelo a linealizar [11].

La TEP posee una serie de incertidumbres que deben manejarse con eficacia, tales como, previsión de la carga, disponibilidad de componentes del sistema de energía y producción de energía; siendo estas, las principales restricciones del sistema [11], [12].

Las LT desempeñan un papel vital en la operación del Sistema, ya que son las encargadas del transporte seguro y confiable de la Energía Eléctrica. Por lo tanto, las adiciones ya sea de generación o de transmisión deben planificarse, lo que implica una gran inversión; por lo que se pretende la construcción de LT con la menor inversión y un grado alto de recuperación del capital. Las pérdidas en LT son de suma importancia, dado que este aspecto representa pérdidas directamente económicas [11], [13]. En consecuencia, la reducción de pérdidas es importante para amenorar costos y obtener niveles de energía óptimos en el sistema.
Para atender el crecimiento de la carga del SEP, la TEP es vital y prioritaria para los planificadores del sistema. En los últimos años, el análisis de los flujos óptimos de potencia (OPF) se ha convertido en una herramienta fundamental para la planificación, operación y control del sistema potencia [11], [14], [16]. El OPF se lo puede analizar de dos maneras, a saber: análisis DC y análisis AC. Cabe resaltar de forma diferencial, que en el caso del análisis DC se desprecian algunas variables del sistema, como son las componentes reactivas. Por tanto, el OPF-AC se establece como un problema de optimización no lineal, con variables discretas y continuas [13].

A partir de los planteamientos anteriormente formulados, se busca mediante la programación entera mixta lineal (MILP), encontrar un equilibrio de la expansión del sistema de transmisión [14]. La función objetivo está basada plenamente en la disminución de pérdidas del sistema para el abastecimiento del SEP, con lo cual se tendrá estabilidad de tensión, la óptima expansión y la mayor confiabilidad del SEP, también hay que tomar en cuenta que todos los elementos del SEP deben operar de manera óptima para ajustarse a las restricciones existentes, balance de potencia activa y reactiva, límites de estabilidad de ángulo, pérdidas en las líneas, balance nodal, entre otras [17], [18]. En consecuencia, se busca obtener un sistema fiable, seguro y de óptima eficiencia para entregar la demanda proyectada [3], [19].

En la presente investigación, se plantea el problema de planificación de la expansión de la transmisión basado en flujos óptimos de potencia linealizados, buscando minimizar los costos asociados a la construcción de nuevas líneas con restricciones, para la reducción de pérdidas técnicas, estabilidad angular, perfil de voltaje nodal y balance de potencia. Cabe resaltar que la linealización de las ecuaciones del flujo de potencia se realiza mediante Series de Taylor, mientras que la optimización se realiza mediante MILP.

En adelante, el artículo se ha estructurado de la siguiente manera: en la sección 2 , se explica sobre la expansión de los sistemas de transmisión; en la sección 3 , se estudia la linealización de los flujos óptimos de potencia; en la sección 4, se analiza el problema de la expansión de los sistemas de transmisión; en la sección 5, se explica la metodología utilizada para el presente estudio; en la sección 6 , se analizan los resultados obtenidos; y finalmente en la sección 7 , se formulan las principales conclusiones.

\section{Expansión de un Sistema de Transmisión}

La expansión de sistemas de transmisión es de gran importancia, dado que se enfoca en garantizar los servicios energéticos para la proyección de una demanda futura y en este sentido, mediante la TEP se desarrolla un algoritmo capaz de tener un modelo eficaz, ya que tiene un mayor impacto para resolver problemas complejos del TEP [2], [14]. 


\section{A. Planificación de la Expansión}

La planificación de las LT debe emprenderse con la suficiente anticipación necesaria, para el posterior inicio de las actividades de construcción, dado que el marco de tiempo para la TEP se puede dividir en términos largos, medios y cortos. La planificación a largo plazo, se realiza para identificar las exigencias técnicas para las nuevas instalaciones de LT y desarrollar nuevas tecnologías desde una perspectiva de la región de suministro. La planificación a mediano plazo, se concentra en los programas de expansión en LT tiene en cuenta un sistema interconectado. La planificación a corto plazo, se realiza para analizar las limitaciones operativas y las soluciones para mantener la continuidad del suministro de energía de calidad a los consumidores [20] requiring a huge amount of OPF (optimal power flow.

Los diversos aspectos técnicos, económicos y ambientales, han sido considerados en la metodología correspondiente a la planificación de la transmisión, teniendo en cuenta ciertos puntos clave para el desarrollo de la metodología de la TEP [21], [22], a saber: evaluación económica, de parámetros, técnica y evaluación del impacto ambiental que este producirá [10].

Diferentes estudios plantean que, para tener una correcta planificación del SEP, es necesario garantizar que la energía sea transportada, con altos niveles de confiabilidad, sin dejar de lado el menor costo posible de inversión en la TEP. En la literatura, se utiliza el sistema de prueba de 3 barras del IEEE y el modelo de Garver [15], [17], [23], [24],[25], los cuales se explican a continuación.

\section{B. Modelo Garver}
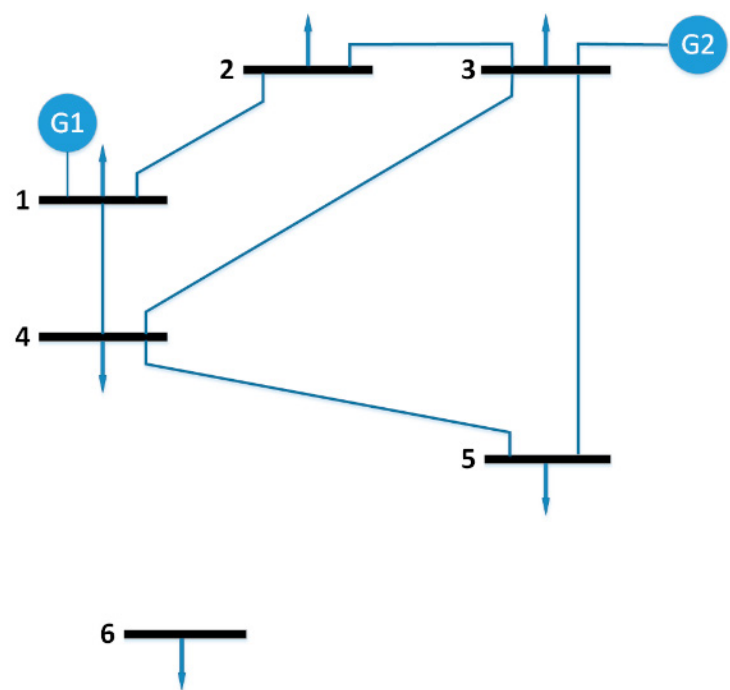

Fig. 1. Sistema de 6 buses con proyección a expansión. Fuente: Autores.
En el esquema se muestra un sistema compuesto por 2 generadores de similares potencias, con 5 cargas conectadas a la red y una carga con proyección a la expansión; como se observa en la Fig. 1, las posibles líneas candidatas a conectar al esquema del LOPF-AC que se observó. El sistema de potencia existente se debe expandir a una condición futura de una carga o generación ya sea el caso, y debe cumplir con el mínimo costo posible de inversión en las líneas candidatas a implementarse teniendo en cuenta que depende de dos factores clave para la inversión que son; el factor de recuperación de capital y la longitud de cada línea candidata a conectarse a la red [17], [26].

\section{III.TÉCNICA PARA LINEALIZAR el Flujo de Potencia AC}

En esta sección se analiza el medio para hacer el ajuste técnico a las ecuaciones que conforman las restricciones del problema de LOPF-AC. Por lo que este método convierte las ecuaciones no lineales de Flujo de Potencia Convencional (AC) en aproximación de ecuaciones lineales. En consecuencia, se procede a desarrollar una aproximación, mediante Series de Taylor de las funciones cuadráticas y de sus funciones sinusoidal y cosenoidal, para modelarlas de forma, que se puedan utilizar a su vez para calcular el LOPF-AC [26], [27], [28].

El proceso de linealizar en este documento, involucra algunas técnicas para el desarrollo lineal, los cuales son: Series de Taylor y Aproximación Lineal mediante derivadas [14], [18]. El LOPF-AC tiene muchas aplicaciones, por lo que se realizará el análisis en la expansión de la transmisión, para el caso se hace la aproximación lineal mediante series de Taylor, un método muy aproximado como se muestra en la Fig. 2, para que exista una operación confiable y segura del sistema [18], [26].

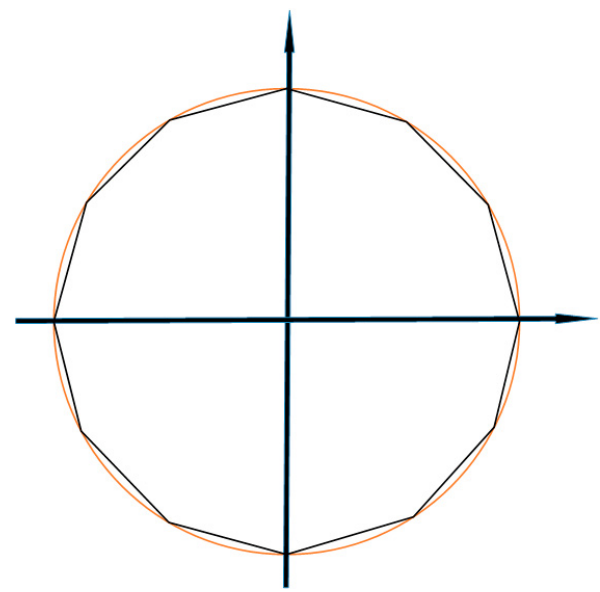

Fig. 2. Aproximación lineal de circulo. Fuente: Autores. 
Para el estudio y análisis del OPF-AC se debe tener claro que no es igual que el análisis en DC, dado que en los estudios TEP, un modelo DC sin pérdidas generalmente se basa en el supuesto según el cual, las pérdidas de línea son limitadas y pueden ignorarse [3], [29]. Sin embargo, esta suposición puede ser problemática para el desarrollo de los estudios de planificación a largo plazo, donde las pérdidas de línea pueden desempeñar un papel en un tiempo determinado largo (es decir, 20 años), [1], [28], y en tal sentido, generar como resultado una planificación de expansión de la red distinta; por lo tanto, se realizará el ajuste de pérdidas de cada línea que conforma el modelo de Garver [17], basándose en los parámetros de la LT como se explica en la Fig. 3.

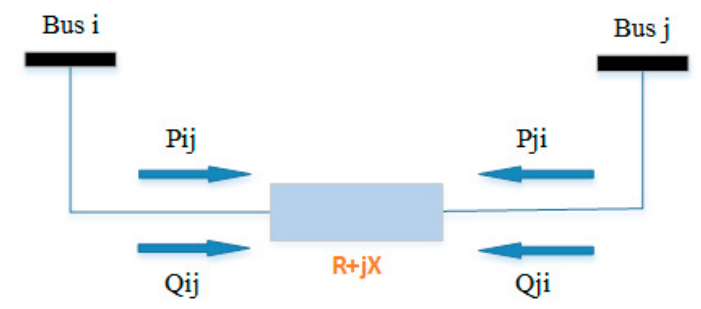

Fig. 3. Esquema de pérdidas del sistema. Fuente: Autores.

\section{Aproximación Lineal}

mediante Series de Taylor

A continuación, aplicamos Series de Taylor para la función cosenoidal y se tiene la siguiente aproximación; el ángulo viene dado en radianes.

$$
\cos \theta_{i j} \approx 1-\frac{\theta_{i j}^{2}}{2}
$$

Se realiza la aproximación de la función sinusoidal con el mismo método de Series de Taylor.

$$
\sin \theta_{i j} \approx 0+\frac{1}{1 !} \theta_{i j}+\frac{0}{2 !} \theta_{i j}^{2}
$$

En consecuencia, la aproximación de la función sinusoidal es la siguiente: el ángulo viene dado en radianes.

$$
\sin \theta_{i j} \approx \theta_{i j}
$$

En este caso, la función de Potencia Activa viene dada por la ecuación.

$$
P_{i j}=\left|V_{i j}\right|^{2} G_{i j}+\left|V_{i}\right|\left|V_{j}\right|\left(G_{i j} \cos \theta_{i j}+B_{i j} \sin \theta_{i j}\right)
$$

Para el modelo propuesto (LOPF-AC) se asume que es un modelo real; para mantener la seguridad y fiabilidad, se debe tener claro las siguientes suposiciones [13], [18]:

- El ángulo $\theta_{i j}$ para cada bus i y j que están conectados por la línea ij es lo suficientemente pequeño.

- La magnitud del voltaje es de aproximadamente 1 p.u. para todos los buses de la Red.

Estas suposiciones son prácticamente verdaderas bajo condiciones normales de operación. Sobre la base de estos supuestos, se propone reescribir mediante la sustitución de las funciones sinusoidales y cosenos con su expansión de la serie Taylor [18], [30].

$$
P_{i j} \approx G_{i j}+\left(G_{i j}\left(1-\frac{\theta_{i j}{ }^{2}}{2}\right)+B_{i j} \theta_{i j}\right)
$$

Por lo tanto, la ecuación que describe a la Potencia Activa está compuesta por parámetros de la Línea de Transmisión y se tendrá lo siguiente.

$$
P_{i j} \approx \frac{1}{2} G_{i j} \theta_{i j}^{2}+B_{i j} \theta_{i j}
$$

Dado que se analizará un LOPF-AC, en este caso, también se debe realizar el estudio de las pérdidas del sistema, que a su vez se puede observar en un circuito simple como en la Fig. 2, el cual se ajusta a los parámetros de cada línea de transmisión. Dicho esto se tiene el siguiente modelo de pérdidas:

$$
P_{e}=P_{i j}+P_{j i}
$$

En esta instancia, se desarrolla la ecuación propuesta mediante las ecuaciones vistas de Potencia Activa.

$$
P_{e}=G_{i j}\left(V_{i}^{2}+V_{j}^{2}-2 V_{i} V_{j} \cos \theta_{i j}\right)
$$

Se puede simplificar de la siguiente manera y se tiene un óptimo ajuste para las pérdidas del sistema.

$$
P_{e}=G_{i j} \theta_{i j}^{2}
$$

\section{Problema de la Expansión de un Sistema de Transmisión Mediante Lopf-Ac}

En esta sección, se procede a estructurar el estándar que tiene un enfoque central del modelo, que está determinado por la minimización de pérdidas en LT, mediante la programación lineal entera mixta (MILP), sujeto a varias restricciones las cuales se verán en dicha sección, para lo cual se tiene la función objetivo a minimizar [4]. 
Tabla 1. Nomenclatura de la Expansión del Sistema de Transmisión.

\begin{tabular}{|c|c|}
\hline & Nomenclatura \\
\hline $\mathrm{i}, \mathrm{j}$ & Índice de cada barra \\
\hline $\mathrm{Li}$ & Índice de líneas \\
\hline $\mathrm{g}$ & Índice de Generadores \\
\hline ex & Índice de existentes \\
\hline $\mathrm{cd}$ & Índice de candidatas \\
\hline Bus & Conjunto de barras \\
\hline Gen & Conjunto de Generadores \\
\hline Lex & Conjunto Líneas existentes \\
\hline Led & Conjunto líneas candidatas \\
\hline Lec & Líneas existentes conectadas \\
\hline Lcdc & Líneas candidatas conectadas \\
\hline$P_{g e n}$ & Potencia Activa de generación \\
\hline$P_{D}$ & Demanda de Potencia Activa \\
\hline $\mathrm{b}$ & Variable binaria \\
\hline$P_{L i}^{e x}$ & Flujo de Potencia Activa de líneas existentes \\
\hline$P_{L i}^{c d}$ & Flujo de Potencia Activa de líneas candidatas \\
\hline$P_{\text {Gen,g }}^{\min }$ & Potencia Activa mínima del generador \\
\hline$P_{\text {Gen,g }}^{\max }$ & Potencia Activa máxima de generador \\
\hline$V_{i}$ & Magnitud de Voltaje \\
\hline$\theta_{i}$ & Magnitud de ángulo \\
\hline$\theta_{B u s}^{\min }$ & Ángulo mínimo en la barra \\
\hline$\theta_{B u s}^{\max }$ & Ángulo máximo en la barra \\
\hline$B_{i j}^{e x}$ & Susceptancia de cada barra existente \\
\hline$B_{i j}^{c d}$ & Susceptancia de cada barra candidata \\
\hline$G_{i j}^{e x}$ & Conductancia de cada barra existente \\
\hline$G_{i j}^{c d}$ & Conductancia de cada barra candidata \\
\hline$C L_{i}$ & Costo de cada línea candidata \\
\hline$P_{e}$ & Perdidas del sistema \\
\hline $\lg _{i}$ & Longitud de línea \\
\hline $\mathrm{A}$ & Parámetros de las barras que compone el sistema \\
\hline B & Parámetros de líneas existentes \\
\hline $\mathrm{C}$ & Parámetros de líneas candidatas \\
\hline $\mathrm{D}$ & Parámetros del generador \\
\hline $\mathrm{Y}$ & Matriz de Admitancia \\
\hline $\mathrm{P}$ & Potencia Activa \\
\hline$P_{e}$ & Pérdidas del Sistema \\
\hline
\end{tabular}

Fuente: Autores

\section{A. Planteamiento del Sistema Eléctrico de Potencia}

El esquema a usar es el modelo de Garver, compuesto por 5 barras, un total de 6 líneas, 2 generadores que operan en condiciones normales y 6 cargas totales en la red como se observa en la Fig. 4.

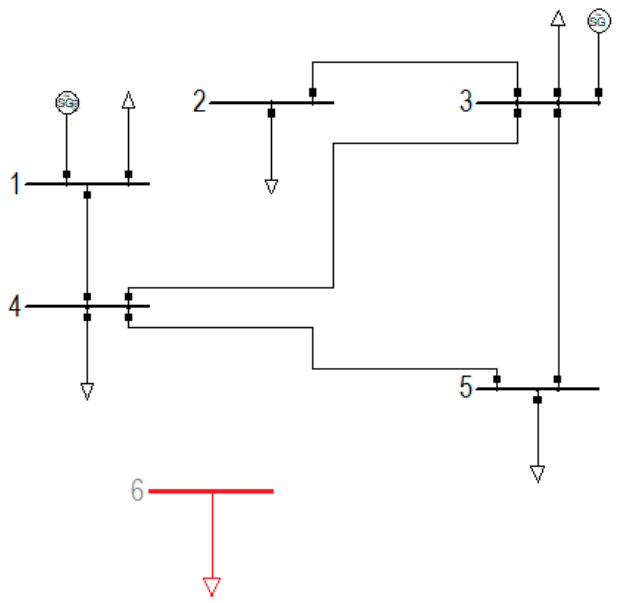

Fig. 4. Modelo de Garver. Fuente: Autores.

\section{B. Formulación del Modelo de Expansión}

Mediante la técnica de LOPF-AC, se tendrá con la función objetivo la minimización de pérdidas de toda la red, tal como se observa en la ecuación (10).

$$
\text { MIN OF }=\sum_{\substack{j=1 \\ i \neq 1}} G_{i j} \theta_{i j}^{2}
$$

La función objetivo debe sujetarse a las siguientes restricciones:

Ahora se procede hacer el balance de Potencia del sistema, con el flujo que circula por cada barra existente. En este caso, la conductancia $G_{i j}$ de la línea entre los nodos del sistema es despreciable en comparación con la susceptancia $B_{i j}$, y en consecuencia se obtiene lo siguiente:

$$
P_{L i}^{e x}=\sum_{\substack{j=1 \\ i \neq 1}}^{n} \frac{1}{2} G_{i j} \theta_{i j}{ }^{2}+B_{i j} \theta_{i j}
$$

Se observa la sumatoria de toda la Potencia Activa que circula en el sistema propuesto de las líneas candidatas que ingresan a la red.

$P_{L i}^{c d}=b \sum_{\substack{j=1 \\ i \neq 1}}^{n} \frac{1}{2} G_{i j} \theta_{i j}{ }^{2}+B_{i j}^{c d} \theta_{i j}$ 
Se observan los límites que restringen la generación de Potencia Activa del sistema.

$P_{G e n}^{\min } \leq P_{G e n} \leq P_{G e n}^{\max }$

De igual forma en (14) se muestran los límites que restringen la diferencia angular del sistema.

$\theta_{i j}^{\min } \leq \theta_{i j} \leq \theta_{i j}^{\max }$

Se muestra en la ecuación (15) de balance de Potencia Activa generada, menos la Potencia demandada del sistema y la Potencia existente del sistema, más la Potencia candidata que circulara por la red.

$$
P_{G e n}-P_{D}=P_{L i}^{e x}+P_{L i}^{c d}
$$

Para la variable binaria se tiene en (16) el máximo de líneas a construir para el sistema.

$$
b \leq N T
$$

\section{Metodología}

Como sistema de potencia a ser analizado se utiliza el modelo de 6 barras de Garver, el cual tiene al nodo 6 como nodo para estudiar la expansión del sistema, dicho nodo no tiene conexión con lo restante del SEP.

En la propuesta, se generó variabilidad de la carga en el nodo 6 y con dicha variabilidad se aplica el TEP planteado, basado en LOPF-AC con restricciones de pérdidas técnicas, se considera que si se dispone de la capacidad de generación requerida por la demanda del sistema; por lo que no se realiza un plan de expansión de la generación.

Se plantean posibles líneas de transmisión paralelas a las existentes, con las mismas características y posibles conexiones desde el nodo 6 hacia cada uno de los nodos existentes; la longitud es seleccionada de manera aleatoria, para que cada futura línea de transmisión tenga diferentes características de impedancia y por ende de admitancia. Para todo el SEP, se utiliza el mismo tipo de conductor con la misma distribución geométrica de las líneas de transmisión.

Se aplica el algoritmo de TEP en el cual se realiza la optimización de los flujos de potencia linealizados (LOPF-AC), minimizando los costos y las pérdidas; respetando el balance de Potencia Activa y reactiva del sistema, límite de estabilidad angular y tensión nodal. Luego de aplicar el algoritmo, se tienen las variables de decisión para seleccionar cuales son las líneas candidatas a ser implementadas, con el propósito de cumplir satisfactoriamente frente a los requerimientos técnicos del sistema.
Tabla 2. Algoritmo para la TEP.

Inicio
Paso 1: Ingreso de los parámetros del sistema.
$A=\left[V, P_{D}, \theta_{i j}^{\min }, \theta_{i j}, \theta_{i j}^{\max }\right]$
$B=\left[N_{i}, N_{j}, R, X\right]$
$C=\left[N_{i}, N_{j}, R, X, l g_{i}, C L_{i}\right]$
$D=\left[N, P, P_{g}^{\min }, P_{g}^{\text {max }}\right]$

Paso 2: Calculo de la conductancia y susceptancia.

$G_{i j}=\frac{R_{i j}}{R_{i j}^{2}+X_{i j}^{2}} ; \quad \forall L i \in L e x^{\wedge} L c d$

$B_{i j}=\frac{X_{i j}}{R_{i j}^{2}+X_{i j}^{2}} ; \quad \forall L i \in L e x^{\wedge} L c d$

Paso 3: Cálculo de la Matriz de Admitancia.

$Y(B, C)=\left[G_{i j}, B_{i j}\right]$

Paso 4: Cálculo del flujo de Potencia Activa de las líneas existentes y candidatas.

$P_{L i}^{e x}=\sum_{\substack{j=1 \\ i \neq 1}}^{n} \frac{1}{2} G_{i j} \theta_{i j}{ }^{2}+B_{i j} \theta_{i j} ; \quad \forall L i \in L e x^{\wedge} L c d$

$P_{L i}^{c d}=b \sum_{\substack{j=1 \\ i \neq 1}}^{n} \frac{1}{2} G_{i j} \theta_{i j}{ }^{2}+B_{i j}^{c d} \theta_{i j} ; \forall L i \in L e x^{\wedge} L c d$

Paso 5: Verificación de los límites de Potencia Activa, de variación de ángulo.

$P_{G e n}^{\min } \leq P_{G e n} \leq P_{G e n}^{\max }$

$\theta_{i j}^{\min } \leq \theta_{i j} \leq \theta_{i j}^{\max }$

Paso 6: Cálculo de Perdidas de por LT.

$P_{e}=G_{i j} \theta_{i j}^{2}$

Paso 7: Verificación del balance de Potencia Activa.

$P_{G e n}-P_{D}=\sum_{i}^{n} P_{L i}^{e x}+\sum_{i}^{n} P_{L i}^{c d}$

Paso 8: Cálculo de las pérdidas totales.

$\sum_{j=1} G_{i j} \theta_{i j}^{2}$

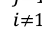

Fin 


\section{Resultados}

Se analizó la sensibilidad de carga en el nodo 6 para la proyección de demanda en el modelo de Garver teniendo los siguientes casos de estudio; como se observa en la tabla 3 .

Tabla 3. Casos de Estudio.

\begin{tabular}{|c|c|}
\hline Casos & Carga $[\mathrm{MW}]$ \\
\hline Caso 1 & 50 \\
\hline Caso 2 & 80 \\
\hline Caso 3 & 110 \\
\hline Caso 4 & 140 \\
\hline Caso 5 & 190 \\
\hline
\end{tabular}

Fuente: Autores

La tabla 4 muestra como mediante LOPF-AC la Potencia Activa generada es necesaria para que exista el balance con la demanda de la red en cada caso de estudio. Al igual que las pérdidas de toda la red, luego de que entra en funcionamiento, dichas LT son candidatas para obtener un sistema fiable y seguro en condiciones de operación nominales.

Tabla 4. Análisis de Despacho de Potencia Activa.

\begin{tabular}{|c|c|c|c|}
\hline Casos & $\begin{array}{c}\text { Potencia Activa } \\
\text { generada [MW] }\end{array}$ & $\begin{array}{c}\text { Pérdidas } \\
{[\mathrm{MW}]}\end{array}$ & $\begin{array}{c}\text { Demanda } \\
{[\mathrm{MW}]}\end{array}$ \\
\hline Caso 1 & 253,7757 & 13,7757 & 240 \\
\hline Caso 2 & 285,8988 & 15,8988 & 270 \\
\hline Caso 3 & 321,9338 & 21,9338 & 300 \\
\hline Caso 4 & 355,3683 & 25,3683 & 330 \\
\hline Caso 5 & 408,8927 & 28,8927 & 360 \\
\hline
\end{tabular}

Fuente: Autores.

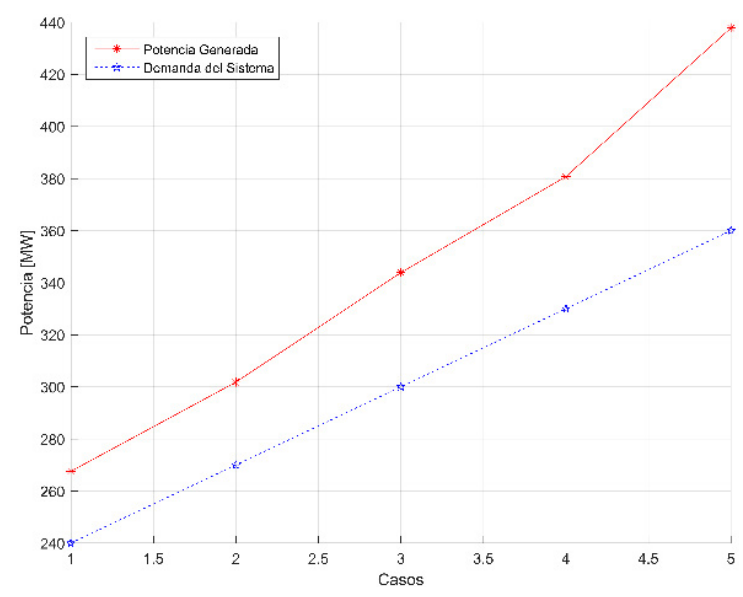

Fig. 5. Comportamiento de Potencia Activa y la proyección de demanda. Fuente: Autores
Se puede observar en la Fig. 5 como el comportamiento de la Potencia Activa para cada caso de estudio, tiene un crecimiento considerable, en la medida en que la demanda lleva un crecimiento lineal dentro del sistema, causando la generación de una mayor potencia activa, para poder contar así con un sistema confiable en la red.

Se ilustra en la Fig. 6 mediante diagrama de barras, el despacho de generación que se realiza en cada caso y se comprueba que la demanda es menor que lo generado; por lo tanto, la diferencia corresponde a las pérdidas totales que existen en cada caso.

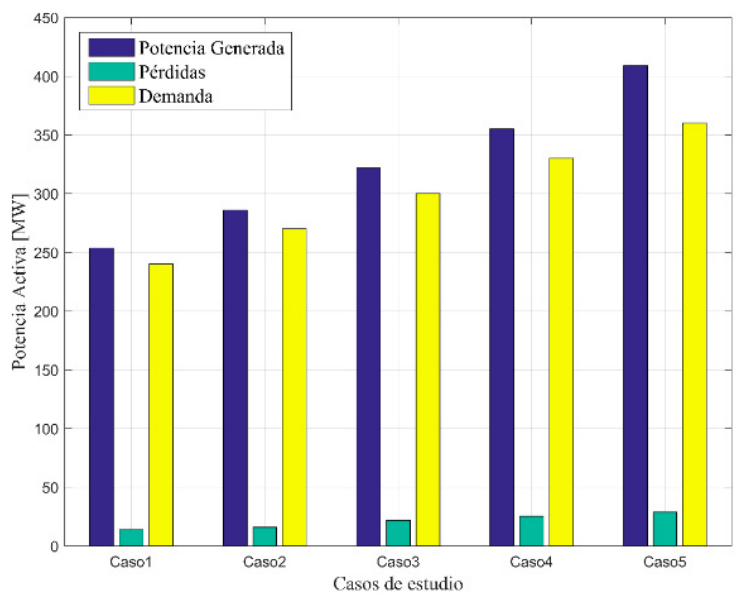

Fig. 6. Despacho de Potencia Activa para cada caso de estudio. Fuente: Autores.

La Fig. 7 muestra el comportamiento del porcentaje de pérdidas en función de la Potencia Activa generada, teniendo el ingreso de posibles LT al sistema ya sea para el reforzamiento o la expansión que se tuvo para los diferentes casos de estudio.

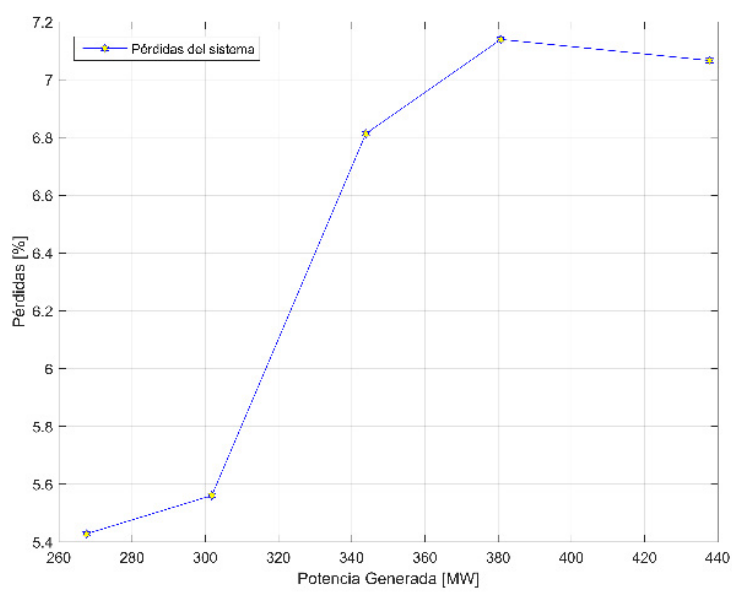

Fig. 7. Porcentaje de pérdidas en función de la Potencia Activa generada. Fuente: Autores.

A continuación, se observa en la tabla 5, los valores que han sido derivados de la simulación de los posibles casos, dependiendo de la proyección de carga en 
el nodo 6; generando a su vez, las siguientes matrices de conexión para las LT candidatas a ingresar a la red, con el fin de conseguir la minimización de pérdidas en todo el sistema y cumplir con el reforzamiento y expansión, para tener un modelo confiable a la red.

Tabla 5. Número de LT a Construir Mediante Análisis de Matriz Binaria.

\begin{tabular}{|c|c|c|c|c|c|c|c|c|c|}
\hline Casos & \multicolumn{7}{|c|}{ Matriz binaria } & $\mathrm{LT}$ & Pérdidas \\
\hline 1 & $\begin{array}{l}\mathrm{n} 1 \\
\mathrm{n} 2 \\
\mathrm{n} 3 \\
\mathrm{n} 4 \\
\mathrm{n} 5 \\
\mathrm{n} 6\end{array}$ & $\begin{array}{c}\mathrm{n} 1 \\
0 \\
0 \\
0 \\
0 \\
0 \\
0\end{array}$ & $\begin{array}{l}\mathrm{n} 2 \\
0 \\
0 \\
0 \\
0 \\
0 \\
1\end{array}$ & $\begin{array}{c}\text { n3 } \\
0 \\
0 \\
0 \\
0 \\
0 \\
0\end{array}$ & $\begin{array}{c}\mathrm{n} 4 \\
0 \\
0 \\
0 \\
0 \\
0 \\
0\end{array}$ & $\begin{array}{c}\text { n5 } \\
0 \\
0 \\
0 \\
0 \\
0 \\
0\end{array}$ & $\begin{array}{c}\mathrm{n} 6 \\
0 \\
1 \\
0 \\
0 \\
0 \\
0\end{array}$ & 1 & 5,428 \\
\hline 2 & $\begin{array}{l}\text { n1 } \\
\text { n2 } \\
\text { n3 } \\
\text { n4 } \\
\text { n5 } \\
\text { n6 }\end{array}$ & $\begin{array}{c}\mathrm{n} 1 \\
0 \\
0 \\
0 \\
0 \\
0 \\
0\end{array}$ & $\begin{array}{l}\mathrm{n} 2 \\
0 \\
0 \\
1 \\
0 \\
0 \\
1\end{array}$ & $\begin{array}{c}\text { n3 } \\
0 \\
1 \\
0 \\
0 \\
0 \\
0\end{array}$ & $\begin{array}{c}\mathrm{n} 4 \\
0 \\
0 \\
0 \\
0 \\
0 \\
0\end{array}$ & $\begin{array}{c}\text { n5 } \\
0 \\
0 \\
0 \\
0 \\
0 \\
0\end{array}$ & $\begin{array}{c}\text { n6 } \\
0 \\
1 \\
0 \\
0 \\
0 \\
0\end{array}$ & 2 & 5,561 \\
\hline 3 & $\begin{array}{l}\mathrm{n} 1 \\
\mathrm{n} 2 \\
\mathrm{n} 3 \\
\mathrm{n} 4 \\
\mathrm{n} 5 \\
\mathrm{n} 6\end{array}$ & $\begin{array}{c}\mathrm{n} 1 \\
0 \\
0 \\
0 \\
0 \\
0 \\
0\end{array}$ & $\begin{array}{c}\mathrm{n} 2 \\
0 \\
0 \\
1 \\
0 \\
0 \\
1\end{array}$ & $\begin{array}{c}\text { n3 } \\
0 \\
1 \\
0 \\
0 \\
0 \\
0\end{array}$ & $\begin{array}{c}\mathrm{n} 4 \\
0 \\
0 \\
0 \\
0 \\
0 \\
1\end{array}$ & $\begin{array}{c}\text { n5 } \\
0 \\
0 \\
0 \\
0 \\
0 \\
0\end{array}$ & $\begin{array}{c}\mathrm{n} 6 \\
0 \\
1 \\
0 \\
1 \\
0 \\
0\end{array}$ & 3 & 6,813 \\
\hline 4 & $\begin{array}{l}\mathrm{n} 1 \\
\mathrm{n} 2 \\
\mathrm{n} 3 \\
\mathrm{n} 4 \\
\mathrm{n} 5 \\
\mathrm{n} 6\end{array}$ & $\begin{array}{c}\mathrm{n} 1 \\
0 \\
0 \\
0 \\
0 \\
0 \\
0\end{array}$ & $\begin{array}{c}\mathrm{n} 2 \\
0 \\
0 \\
0 \\
0 \\
0 \\
1\end{array}$ & $\begin{array}{c}\mathrm{n} 3 \\
0 \\
0 \\
0 \\
0 \\
0 \\
1\end{array}$ & $\begin{array}{c}\mathrm{n} 4 \\
0 \\
0 \\
0 \\
0 \\
0 \\
0\end{array}$ & $\begin{array}{c}\mathrm{n} 5 \\
0 \\
0 \\
0 \\
0 \\
0 \\
0\end{array}$ & $\begin{array}{c}\mathrm{n} 6 \\
0 \\
1 \\
1 \\
0 \\
0 \\
0\end{array}$ & 2 & 7,139 \\
\hline 5 & $\begin{array}{l}\mathrm{n} 1 \\
\mathrm{n} 2 \\
\mathrm{n} 3 \\
\mathrm{n} 4 \\
\mathrm{n} 5 \\
\mathrm{n} 6\end{array}$ & $\begin{array}{c}\mathrm{n} 1 \\
0 \\
0 \\
0 \\
0 \\
0 \\
0\end{array}$ & $\begin{array}{c}\mathrm{n} 2 \\
0 \\
0 \\
1 \\
0 \\
0 \\
1\end{array}$ & $\begin{array}{c}\text { n3 } \\
0 \\
1 \\
0 \\
0 \\
0 \\
1\end{array}$ & $\begin{array}{c}\mathrm{n} 4 \\
0 \\
0 \\
0 \\
0 \\
0 \\
0\end{array}$ & $\begin{array}{c}\text { n5 } \\
0 \\
0 \\
0 \\
0 \\
0 \\
1\end{array}$ & $\begin{array}{c}\mathrm{n} 6 \\
0 \\
1 \\
1 \\
0 \\
1 \\
0\end{array}$ & 4 & 7,066 \\
\hline
\end{tabular}

Fuente: Autores.
Para cada caso de estudio, se ha determinado un plan de expansión diferente, como se puede apreciar en la tabla 5. Para el caso de estudio (1), en el cual, la carga demandada en el nodo 6 es de $50 \mathrm{MW}$, se requiere crear una línea de transmisión que conecte al nodo 6 con el nodo 2. Para el caso 2, cuando la carga es de $80 \mathrm{MW}$, se requieren dos líneas de transmisión nuevas que conecten al nodo 6 con el nodo 2 y el nodo 3 con el nodo 2 . En consecuencia, se requiere aumentar un circuito de conexión entre dichos nodos.

Para el caso 3 , se requiere crear tres líneas de transmisión nuevas que conecten al nodo 6 con el nodo 2 , nodo 6 con el nodo 4 y el nodo 3 con el nodo 2 , para poder suplir a la carga de $110 \mathrm{MW}$ que estará conectada al nodo 6. Para el caso 4 donde la demanda en el nodo 6 es de $140 \mathrm{MW}$ se requiere dos líneas de transmisión nuevas que conecte al nodo 6 con el nodo 2 y el nodo 6 con el nodo 3 .

Para el caso de estudio 5, donde la demanda en el nodo 6 es de $190 \mathrm{MW}$, se requiere cuatro líneas de transmisión nuevas que conecten al nodo 6 con el nodo 2 , nodo 6 con el nodo 3 , nodo 6 con el nodo 5 y nodo 3 con el nodo 2; tal como se puede apreciar en la Fig. 8. Para este caso las pérdidas de potencia en las líneas de transmisión son de $7.06 \%$.

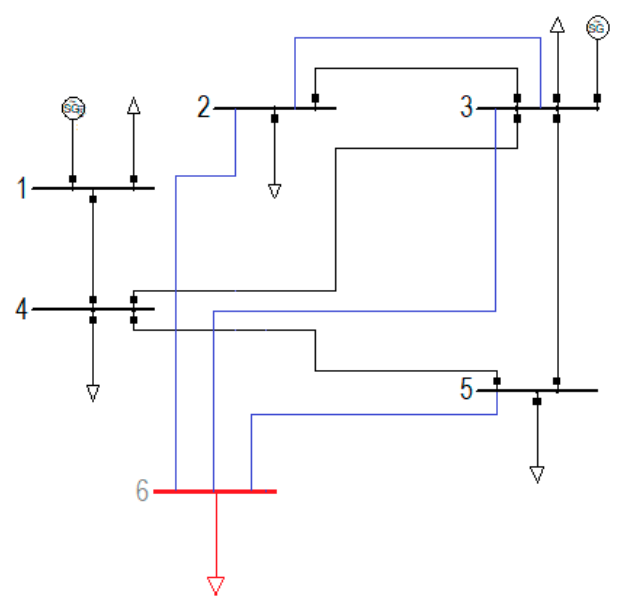

Fig. 8. Expansión del sistema de transmisión. Fuente: Autores.

\section{Conclusiones y Recomendaciones}

La demanda total de la red unida con la proyección de carga de los distintos casos para la expansión del sistema de transmisión, es abastecida con normalidad, cumpliendo con los parámetros establecidos de generación y transmisión, conjuntamente con las restricciones del algoritmo, para efecto de obtener un desempeño óptimo en la TEP.

La metodología para la TEP, consta de dos variables claves que son la longitud de las LT y el costo de construcción de dichas LT; en consecuencia, el LOPFAC encontró un punto seguro y preciso, como solución posible para obtener el costo de inversión y pérdidas mínimas en todo el sistema para la TEP. 
Mediante la aproximación del LOPF-AC, se desarrolló un manejo simple de las ecuaciones, tanto para la función objetivo, como las restricciones del sistema de igualdad y desigualdad, más los límites de variables del proceso; para tener resultados óptimos del SEP, con el fin de poder realizar diferentes casos de estudio.

El flujo óptimo de Potencia AC convencional, es un problema matemático más robusto por lo cual de difícil solución. Si es analizado en el software GAMS, presentará problemas de convergencia, dado que depende del tamaño del SEP; mientras que el LOPF-AC, permite dar una solución óptima en un punto viable de operación del SEP, tomando en cuenta solo la Potencia Activa de todo el sistema, minimizando los datos de elementos y restricciones del algoritmo.

\section{REFERENCIAS}

[1] S. S. Taheri, J. Kazempour and S. Seyedshenava, "Transmission expansion in an oligopoly considering generation investment equilibrium," Energy Econ., vol. 64, pp. 5562, 2017. https://doi.org/10.1016/j.eneco.2017.03.003

[2] T. Akbari, A. Rahimi-kian and M. Heidarizadeh, "Security-Constrained Transmission Expansion Planning: A Multi-Objective Approach," lectrical Eng. (ICEE), 2011 19th Iran. Conf., p. 6, 2011

[3] D. Sainju, R. Sinha and B. R. Pokhrel, "Static Expansion Planning of Transmission Line Using Mixed Integer Linear Programming Method," in Power Systems (ICPS), 2016 IEEE 6th International Conference on, 2016, pp. 1-6. https://doi.org/10.1109/ICPES.2016.7584141

[4] P. V. Escudero and D. F. Carrión, "Modelo de Expansión de un sistema de transmisión basado en linealización de flujos de potencia óptimos AC.," p. 34, 2018.

[5] D. Carrión, E. Inga, J. W. Gonzalez and R. Hincapié, "Optimal Geographical Placement of Phasor Measurement Units based on Clustering Techniques," in 51st International Universities' Power Engineering Conference, 2016, p. 6. https://doi.org/10.1109/UPEC.2016.8114003

[6] W. H. Caisapanta and D. F. Carrión, "Expansión de sistemas de transmisión eléctrica usando criterios de óptima potencia AC," 2016.

[7] D. Carrión, J. W. González, I. A. Isaac and G. J. López, "Optimal Fault Location in Transmission Lines Using Hybrid Method," in 2017 IEEE PES Innovative Smart Grid Technologies Conference, 2017, p. 6. https://doi. org/10.1109/ISGT-LA.2017.8126757

[8] G. Yaguana and D. Carrión, "Optimización de la expansión de los sistemas de transmisión usando gams.pdf." Quito, p. 21, 2016

[9] R. Hemmati, R.-A. Hooshmand and A. Khodabakhshian, "Comprehensive review of generation and transmission expansion planning," IET Gener. Transm. Distrib., vol. 7, no. 9, pp. 955-964, Sep. 2013. https://doi.org/10.1049/ iet-gtd.2013.0031

[10] A. K. Ferdavani, M. Salem, I. Alhamrouni and A. Khairuddin, "Transmission expansion planning using AC-based differential evolution algorithm," IET Gener. Transm. Distrib., vol. 8, no. 10, pp. 1637-1644, Oct. 2014. https://doi.org/10.1049/iet-gtd.2014.0001

[11] G. Latorre, R. Dario Cruz, J. M. Areiza and A. Villegas, "Classification of publications and models on transmission expansion planning," IEEE Trans. Power Syst., vol. 18, no. 2, pp. 938-946, 2003. https://doi.org/10.1109/ TPWRS.2003.811168
[12] T. Akbari, A. Rahimi-Kian and M. Tavakoli Bina, "Security-constrained transmission expansion planning: A stochastic multi-objective approach," Int. J. Electr. Power Energy Syst., vol. 43, no. 1, pp. 444-453, 2012. https://doi.org/10.1016/j.ijepes.2012.05.058

[13] J. Marecek, M. Mevissen and J. C. Villumsen, "MINLP in transmission expansion planning," in Power Systems Computation Conference (PSCC), 2016, pp. 1-8. https:// doi.org/10.1109/PSCC.2016.7540906

[14] A. Capasso, A. Cervone, R. Lamedica and L. Palagi, "A LP and MILP methodology to support the planning of transmission power systems," Electr. Power Syst. Res., vol. 140, pp. 699-707, 2016. https://doi.org/10.1016/j. epsr.2016.04.024

[15] M. Jadidoleslam, A. Ebrahimi and M. A. Latify, "Probabilistic transmission expansion planning to maximize the integration of wind power," Renew. Energy, vol. 114, pp. 866-878, 2017. https://doi.org/10.1016/j.renene.2017.07.063

[16] D. Carrión, E. Inga, J. W. Gonzalez, and R. Hincapié, "Optimal Geographical Placement of Phasor Measurement Units based on Clustering Techniques," in 2016 51st International Universities Power Engineering Conference, 2016, pp. 6-11. https://doi.org/10.1109/ UPEC.2016.8114003

[17] L. Garver, "Transmission Network Estimation Using Linear Programming," IEEE Trans. Power Appar. Syst., vol. PAS-89, no. 7, pp. 1688-1697, 1970. https://doi org/10.1109/TPAS.1970.292825

[18] H. Zhang, V. Vittal, G. T. Heydt and J. Quintero, "A relaxed AC optimal power flow model based on a Taylor series," 2013 IEEE Innov. Smart Grid Technol. (ISGT Asia), pp. 1-5, 2013.

[19] D. Carrion, J. W. Gonzalez, I. A. Isaac, G. J. Lopez and H. A. Cardona, "Load Characterization Based on Voltage and Current Phasorial Measurements in Micro-Grids," 2017 Int. Conf. Inf. Syst. Comput. Sci., pp. 1-6, 2017. https://doi.org/10.1109/INCISCOS.2017.23

20] D. Z. Fitiwi, L. Olmos, M. Rivier, F. de Cuadra and I. J. Pérez-Arriaga, "Finding a representative network losses model for large-scale transmission expansion planning with renewable energy sources," Energy, vol. 101, pp. 343-358, 2016. https://doi.org/10.1016/j.energy.2016.02.015

[21] S. de la Torre, A. J. Conejo and J. Contreras, "Transmission expansion planning in electricity markets," IEEE Trans. Power Syst., vol. 23, no. 1, pp. 238-248, 2008. https://doi.org/10.1109/TPWRS.2007.913717

[22] C. A. Sima, G. C. Lazaroiu and V. Dumbrava, "Transmission expansion planning optimization for improving RES integration on electricity market," in 2017 10th International Symposium on Advanced Topics in Electrical Engineering (ATEE), 2017, pp. 855-859. https://doi. org/10.1109/ATEE.2017.7905085

23] M. Tavakoli Bina and T. Akbari, "Approximated MILP model for AC transmission expansion planning: global solutions versus local solutions," IET Gener. Transm. Distrib., vol. 10, no. 7, pp. 1563-1569, 2016. https://doi. org/10.1049/iet-gtd.2015.0723

24] L. P. Garcés, A. J. Conejo, R. García-Bertrand and R. Romero, "A bilevel approach to transmission expansion planning within a market environment," IEEE Trans. Power Syst., vol. 24, no. 3, pp. 1513-1522, 2009. https:// doi.org/10.1109/TPWRS.2009.2021230

[25] G. Srinivasulu, "Multi- Objective Transmission Expansion Planning for IEEE 24 Bus RTS," pp. 144-149, 2015. https://doi.org/10.1109/PCCCTSG.2015.7503895

[26] T. Akbari and M. Tavakoli Bina, "A linearized formulation of AC multi-year transmission expansion planning. A mixed-integer linear programming approach," Electr. Power Syst. Res., vol. 114, pp. 93-100, Sep. 2014. https:// doi.org/10.1016/j.epsr.2014.04.013 
[27] M. Olofsson, G. Andersson and L. Soder, "Linear programming based optimal power flow using second order sensitivities," IEEE Trans. Power Syst., vol. 10, no. 3, pp. 1691-1697, 1995. https://doi.org/10.1109/59.466472

[28] H. Zhang, V. Vittal, G. T. Heydt and J. Quintero, "A Mixed-Integer Linear Programming Approach for Multi-Stage Security-Constrained Transmission Expansion Planning," Power Syst. IEEE Trans., vol. 27, no. 2, pp. 1125-1133, 2012. https://doi.org/10.1109/ TPWRS.2011.2178000

[29] A. Lotfjou, Y. Fu and M. Shahidehpour, "Hybrid AC/DC Transmission Expansion Planning," IEEE Trans. Power Deliv., vol. 27, no. 3, pp. 1620-1628, Jul. 2012. https:// doi.org/10.1109/TPWRD.2012.2194515

[30] N. Alguacil, A. L. Motto, and A. J. Conejo, "Transmission expansion planning: A mixed-integer LP approach," IEEE Trans. Power Syst., vol. 18, no. 3, pp. 1070-1077, 2003. https://doi.org/10.1109/TPWRS.2003.814891
Pablo Escudero Delgado realizó sus estudios secundarios en el Colegio Andino donde obtuvo el título de Bachiller en Física Matemático. Egresado de la carrera de Ingeniería Eléctrica de la Universidad Politécnica Salesiana. Su campo de interés se encuentra relacionado con operación en generación y sistemas de transmisión, protecciones eléctricas, planificación de sistemas de distribución. En la actualidad es miembro del Grupo de Investigación GIREI (Grupo de Investigación en redes Eléctricas InteligentesSmart Grid Research Group).

Diego Carrión Galarza se graduó en Ingeniería Eléctrica de la Universidad Politécnica Salesiana (Ecuador) en 2010 y en la actualidad está trabajando para optar su título de Doctor en Ingeniería de la Universidad Pontificia Bolivariana - Medellín (Colombia). Es profesor e investigador en la Universidad Politécnica Salesiana - Quito (Ecuador). En la actualidad es miembro del Grupo de Investigación GIREI (Grupo de Investigación en redes Eléctricas Inteligentes - Smart Grid Research Group). 